\title{
Network-wise surface-based morphometric insight into the cortical neural circuitry underlying irritability in adolescents
}

\author{
Sahil Bajaj $\mathbb{D}^{1,2}{ }^{凶}$, Karina S. Blair ${ }^{1,2}$, Johannah Bashford-Largo ${ }^{1,2}$, Ru Zhang ${ }^{1,2}$, Avantika Mathur ${ }^{1,2}$, Amanda Schwartz ${ }^{1,2}$, \\ Jaimie Elowsky ${ }^{1,2}$, Matthew Dobbertin ${ }^{1,2}$, Soonjo Hwang ${ }^{3}$, Ellen Leibenluft ${ }^{4}$ and R. James R. Blair ${ }^{1,2}$
}

(c) The Author(s) 2021

Previous studies examining structural brain correlates of irritability have taken a region-specific approach and have been relatively inconsistent. In a sample of adolescents with and without clinically impairing irritability, the current study examines: (i) cortical volume (CV) in canonical functional networks; (ii) the association between the CV of functional networks and severity of irritability; and (iii) the extent to which IQ mediates the association between structural abnormalities and severity of irritability. Structural MRI and IQ data were collected from 130 adolescents with high irritability (mean age $=15.54 \pm 1.83$ years, 58 females, self-reported Affective Reactivity Index $[A R I] \geq 4$ ) and 119 adolescents with low irritability (mean age $=15.10 \pm 1.93$ years, 39 females, selfreported ARI < 4). Subject-specific network-wise CV was estimated after parcellating the whole brain into 17 previously reported functional networks. Our Multivariate Analysis of Covariance (MANCOVA) revealed that adolescents with high irritability had significantly reduced CV of the bilateral control and default-mode networks $(p<0.05)$ relative to adolescents with low irritability. Multiple regression analyses showed a significant negative association between the control network CV and the severity of irritability. Mediation analysis showed that IQ partially mediated the association between the control network CV and the severity of irritability. Follow-up analysis on subcortical volume (SCV) showed that adolescents with high irritability had reduced bilateral SCV within the amygdala relative to adolescents with low irritability. Reduced CV within bilateral control and default networks and reduced SCV within bilateral amygdala may represent core features of the pathophysiology of irritability. The current data also indicate the potential importance of a patient's IQ in determining how pathophysiology related to the control network is expressed.

Translational Psychiatry (2021)11:581; https://doi.org/10.1038/s41398-021-01710-2

\section{INTRODUCTION}

Irritability is the propensity to initiate an angry response to frustration, threat, and social provocation and can be defined as "mood (trait) and behavioral (reactive state) dysregulation" that results in a propensity to react and respond angrily when an individual's ability to attain a goal is blocked [1-3]. It can be clinically concerning when the individual's expression of anger is high in relation to their peers $[4,5]$. Prior work has been successful exploring the focal structural deficits associated with irritability but has limited our ability to better understand the network-wise (i.e., at a larger-scale) structural architecture of the brain underlying irritability. Notably, network-level architecture represents the organizational properties of distributed brain systems based on intrinsic functional connectivity patterns [6]. It's still unclear how altered structural/cortical organizational properties of such distributed functional brain systems are associated with irritability in adolescents. The current study aims to determine the extent to which the network-wise cortical structure of adolescents with high irritability differs from adolescents with low irritability.

Functional neuroimaging work has relatively consistently related irritability to dysfunctions in both response control [7-9] and emotional responding $[10,11]$. However, prior brain morphometry/structural studies have been less consistent with respect to: (i) the extent to which irritability is associated with greater cortical volume/cortical thickening $[12,13]$ or decreased grey matter volume/cortical thinning [14-17]; and (ii) the cortical regions implicated. Thus, among studies reporting that irritability is associated with greater grey matter volume/cortical thickening, one implicated the medial orbitofrontal and cingulum/cingulate cortices [12] and the other one implicated the superior frontal and temporal gyri, and the inferior parietal lobule [13]. Among studies reporting that irritability is associated with decreased grey matter volume/cortical thinning, two implicated the dorsolateral prefrontal cortex $[15,16]$ and two implicated the inferior frontal and temporal cortices $[14,17]$. Thus, cortical alterations only within the frontal and temporal cortices appear to be consistent.

The first two goals of the current study were to determine network-wise cortical volume (CV) differences between adolescents with high irritability and adolescents with low irritability, and to further investigate associations between CV of identified networks (and their component regions) and irritability symptom severity. We measured CV from 17 cortical networks using one of

\footnotetext{
${ }^{1}$ Multimodal Clinical Neuroimaging Laboratory (MCNL), Center for Neurobehavioral Research, Boys Town National Research Hospital, Boys Town, NE, USA. ${ }^{2}$ Program for Trauma and Anxiety (PTAC), Center for Neurobehavioral Research, Boys Town National Research Hospital, Boys Town, NE, USA. ${ }^{3}$ Department of Psychiatry, University of Nebraska Medical Center, Omaha, NE, USA. ${ }^{4}$ Section on Mood Dysregulation and Neuroscience, National Institute of Mental Health, National Institutes of Health, Bethesda, MD, USA.

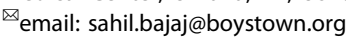


the most advanced analytical methods, which does not over- or under-represent tissue according to the cortical convolutions [18]. Specifically, we used a network-based approach to parcellate the whole brain into 17 standard functional brain networks using Yeo's atlas (see Supplementary Fig. 1 and Supplementary Information) [6]. To our knowledge, Yeo's 17 network atlas is the only atlas that provides a detailed and finest network-wise cortical parcellation implemented in cutting-edge pipelines (e.g., in FreeSurfer toolbox) to quantify cortical morphometry. Prior work has used region-specific cortical parcellations in youth aged 9-10 years [19]; however, this is the first study where 17-network parcellation has been implemented in youth with irritability. While it is true this parcellation was originally developed from adult participants [6], subsequent work has demonstrated that 400 regional parcellation (that was assigned according to 17 network parcellation) reflects the network organization in youth aged 9-10 years [20]. Therefore, determining the CV of these networks and the extent to which they are atypical in individuals with high levels of irritability may improve the interpretability of findings in terms of their association with irritability. Because the primary focus of the current study is on the CV of 17 cortical networks, therefore, in our main analysis, we used $C V$ as our primary parameter to quantify brain morphometry of each of the 17 cortical networks, whereas in follow-up analysis, we repeated our analysis and explored networkwise thickness and surface area, as well as subcortical volume (SCV) of the six main subcortical structures (i.e., thalamus, caudate, putamen, pallidum, hippocampus, and amygdala).

The third goal of this study was to determine the extent to which IQ mediates the association between the network-wise CV and irritability symptom severity. Previous work has shown strong associations between the cortical structure and IQ [21-23]. Specifically, IQ has been positively associated with a cortical thickness within the anterior-ventral prefrontal and superior frontal, superior parietal, and inferior and superior temporal cortices $[22,23]$ all regions implicated in structural MRI studies of irritability [14-17]. Higher IQ has also long been considered a protective factor against the development of mental illness (i.e., individuals with low IQ may be more vulnerable to mental illness) [24-26]. Higher IQ may facilitate the development of functions, such as executive functioning and internal locus of control [27-30] and as such may mediate the relationship between the cortical structure and mental illness. Specifically, the current study will test the extent to which IQ mediates the relationship between CV and irritability.

The goals of the current study were to (i) identify brain networks that show differences in CV between adolescents with high irritability and adolescents with low irritability; (ii) investigate the association between CV of identified brain networks (and their component regions) and irritability; and (iii) determine the extent to which IQ mediates the association between the altered network-wise cortical structure and greater levels of irritability. Given the prior neuroimaging work on irritability and dysfunctions in response control and emotional responding [7-11] and most of the prior structural MRI work showing decreased grey matter volume/cortical thinning associated with irritability [14-17], we predicted that irritability would be associated with reduced CV in networks hypothesized to underpin response control and emotional responding (i.e., the limbic, control, and default-mode networks). Specifically, we hypothesized that: (i) adolescents with high irritability would show lower CV within these networks compared to adolescents with low irritability; (ii) CV within these networks and their component regions would be negatively associated with irritability; and (iii) IQ would mediate the association between the altered cortical structure and irritability. In our follow-up exploratory analysis, we repeated our analysis with thickness and surface area as well as for SCV of six subcortical structures (i.e., thalamus, caudate, putamen, pallidum, hippocampus, and amygdala).

\section{METHODS}

\section{Participants}

The current study included data collected from 249 youths between 11 and 19 years of age $\left(M_{\text {age }}=15.33 \pm 1.89\right.$ years, 97 females). Participants were recruited from a residential care facility at Boys Town National Research Hospital (BTNRH) and from the surrounding community. Participants recruited from the residential facility had been referred for behavioral and emotional problems. Participants were divided into high and low irritability groups based on their irritability scores and were matched across sex and age. Specifically, following guideline from previous work [31], those scoring 4 or greater on the Affective Reactivity Index (ARI) [32] were in the high irritability group $(N=130 ; 72$ males; $M_{\text {age }}=15.54 \pm 1.83$ years; age range: $11-19 ; \mathrm{ARI} \geq 4, M_{A R I}=6.88 \pm 2.52$ ), while those scoring less than 4 were placed in the low irritability group $\left(N=119 ; 39\right.$ females; $M_{\text {age }}=15.10 \pm 1.93$ years; age range: $11-19 ; \mathrm{ARI}<4$; $\left.M_{A R I}=0.84 \pm 0.91\right)$. Consistent with that, receiver operating characteristic (ROC) analysis on the current sample showed that a cut-off value of 3.5 on self-reported ARI was optimal for indicating psychopathology; with both specificity and sensitivity of $100 \%$. Here, all participants scoring ARI $<4$ were recruited from the community and were basically a matched control group. However, the control group was referred as "low irritability group" in the current study because not all these participants scored 0 on the ARI scale but had ARI scores ranged between 0 and 4 . Licensed and boardcertified child and adolescent psychiatrists provided diagnoses following clinical interviews with the participants and their parents to adhere closely to common clinical practice. All participants and their parents provided written informed assent/consent prior to enrollment. The study protocol was approved by the Institutional Review Board at BTNRH. See Section 1 in Supplementary Material for details regarding exclusion/inclusion criteria.

\section{Data collection}

Neuroanatomical data. Structural MRI data were collected using 3-Tesla MRI scanner located at BTNRH. Each participant was instructed repeatedly to try their best to minimize head movement during the entire scan. Custom-made padding was used to minimize head motion. Whole-brain anatomical data for each participant were acquired using a 3D magnetization-prepared rapid acquisition gradient echo (MPRAGE) sequence, which consisted of 176 axial slices (repetition time $=2200 \mathrm{~ms}$, echo time $=2.48 \mathrm{~ms}$, matrix size $=256 \times 208$, slice thickness $=1 \mathrm{~mm}$, voxel resolution $=0.9 \times 0.9 \times 1 \mathrm{~mm}^{3}$, field of view $(F O V)=230 \mathrm{~mm}$, and flip angle $=8^{\circ}$ )

General intelligence (IQ). The two-subset form [i.e., Full-Scale IQ-2 Subtests (FSIQ-2) from FSIQ-4] of the Wechsler Abbreviated Scale of Intelligence II

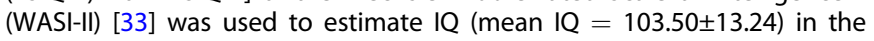
domains of matrix and vocabulary reasoning. The FSIQ-2 scores have a high-reliability coefficient $(a=0.93)$ in juvenile samples (age 6-16 years) [34] and a strong correlation of 0.94 with FSIQ-4 scores [35].

Self-reported affective reactivity index (ARI). The ARI is a seven-item selfreport questionnaire (six symptom items i.e., "I am easily annoyed by others", "I often lose my temper", "I stay angry for a long time", "I am angry most of the time", "I get angry frequently", and "I lose my temper easily" and one function impairment item i.e., "Overall, my irritability causes me problems") that assesses the youth's irritability during the preceding 6 months [32]. Participants were asked to mark the box for "Not True", "Somewhat True" or "Certainly True" corresponding to each item. Prior work has indicated that the ARI is a reliable $(a=0.88$ in the US sample with a mean age of $12.90 \pm 2.70$ years; age range $6-17$ ) and valid measure of irritability in youth [32].

Self-reported measures of other psychopathologies. Psychopathology was indexed via the: (i) Conners ADHD scale [36] to measure the severity of attention deficit/hyperactivity disorder symptoms; (ii) Inventory of CallousUnemotional Traits (ICU) [37] to assess callous-unemotional traits (CU); (iii) Mood and Feeling Questionnaire (MFQ) [38] to assess depression symptomatology; and (iv) Generalized Anxiety Disorder (GAD) subscale using the Screen for Child Anxiety Related Disorders (SCARED) [39] to assess anxiety symptoms.

\section{Image preprocessing}

Recent morphometry methods have allowed researchers to estimate several cortical measures, including CV, cortical thickness, and cortical surface area. As CV is the product of cortical thickness and cortical surface 
area, both cortical thickness and cortical surface area measurements influence CV measurements [40]. While the analysis of the CV (i.e., joint analysis of thickness and surface area) may be potentially more informative as it increases the power to simultaneously quantify the effects of thickness and surface area; however, analyzing thickness and surface area individually may also improve the specificity compared to CV. In our primary analysis, we used $C V$ as our main parameter to quantify brain morphometry, whereas in follow-up analysis, we repeated our analysis and explored network-wise thickness and surface area as well. The "recon-all" pipeline from the FreeSurfer toolbox (Version 6.0; https://surfer.nmr.mgh. harvard.edu) was used to process the structural brain images [41, 42] and for estimating CV, thickness, and surface area measures. Processing of structural images involved basic image preprocessing steps, including head motion-correction, brain extraction (i.e., removal of nonbrain tissue), automated transformation to the standard MNI template space, volumetric segmentation into cortical and sub-cortical matter, intensity correction, and parcellation of the cerebral cortex into gyral and sulcal matter [43]. The technical details of the preprocessing steps are documented in previous publications [41, 42, 44]. To inspect the preprocessing accuracy, standard quality control steps were performed. These steps included a careful visual inspection of raw structural images, skull-stripped brain volumes, and pial surfaces.

\section{Data analysis}

Outlier detection. Age, IQ, and ARI scores data were screened for outliers using SPSS 25 (https://www.ibm.com/analytics/spss-statistics-software) Any participants with a value (on either age, IQ, or ARI scores) more than 1.5 inter-quartile range above/below the upper/lower quartile were identified as outliers and were excluded from our analysis.

Demographics characteristics. Potential group differences in sex were examined via Chi-squared tests while those for age, IQ, and intracranial volume (ICV) were examined via two samples $t$-tests using SPSS 25.

Network-wise group differences in CV. The measure of CV (i.e., the amount of grey matter that lies between white-grey matter interface and pial matter) [40, 45] was evaluated separately for the left and the right hemisphere for each individual. Yeo's Atlas [6] was used to estimate the CV of 17 functional brain networks for each hemisphere (for more details about these networks, see Supplementary Fig. 1 and Supplementary Information). For the between-group/main effect and between subjecteffect analysis, hemispheric-wise CV data were compared between adolescents with high irritability and adolescents with low irritability using multivariate analysis of covariance (MANCOVA; with sex, age, IQ, and ICV as covariates) in SPSS 25. Only the networks that showed significant group differences (at $p<0.05$ ) bilaterally (i.e., for both left and right hemispheres) were considered for further analysis. Multiple comparison correction was not performed across 17 networks because it is generally agreed that if MANCOVA shows a significant group effect, it is important to understand what components (in this case networks) are driving this group effect.

Statistical assumptions: Prior to conducting MANCOVA, CV data were tested for normality (using skewness and kurtosis) and homogeneity of the covariance (using Levene's test of error variances) in SPSS 25. Data is considered to (a) be normal if skewness is ranged between -2 and +2 and kurtosis is between -7 and +7 [46], and (b) meet the assumption of homogeneity of the covariance if Levene's test is not statistically significant. The current analysis showed that hemispheric-wise CV data followed a normal distribution with skewness ranged between -1 and +1 and kurtosis ranged between -1.1 and +1.1 , and that the equality of variance assumption is true $(p>0.05)$ for 28 (out of 34 ) hemispheric-wise CV data points. Moreover, ARI data also followed a normal distribution with skewness and kurtosis magnitude of 0.61 and -0.69 , respectively.

Associations between mean CV and irritability symptom severity. CV data were averaged over hemispheres for each identified network (i.e., the networks that bilaterally showed significant group differences). Dimensional analyses tested the association between hemispheric mean CV of the identified networks and irritability scores across the full sample. These analyses involved multiple regression with covariates (i.e., sex, age, IQ, and ICV) and mean CV of identified networks to potentially predict irritability scores.

Associations between mean region-specific $\mathrm{CV}$ and irritability symptom severity. CV data were averaged over hemispheres for all the component regions of each identified network that showed significant association with irritability. Here, component regions within each of the identified networks were extracted using aparc.annot (Desikan-Killany Atlas) [43], while the CV of these regions was extracted using the mri segstats pipeline from FreeSurfer. Pearson's partial correlation analyses (with sex, age, IQ, and ICV as covariates) were conducted to determine associations between hemispheric mean CV of component regions of the identified networks and irritability scores. Data points with Cook's distance of more than four times the mean were considered as outliers and were excluded from the analysis. All the above analyses were conducted in MATLAB R2021a.

Mediation analysis: role of IQ in mediating the association between CV and irritability symptom severity. Separate standard mediation analyses (model 4) with 10,000 bootstrap samples were conducted using the Hayes PROCESS macro program [47] in SPSS 25 to examine the significance of indirect effects (at 95\% confidence intervals) i.e., to determine the extent at which IQ mediates the association of (a) hemispheric mean CV of identified networks and (b) their component regions (i.e., networks/regions that showed significant association with irritability) with irritability. Because age and sex were not significant confounds, we did not include them in our mediation analysis. Data were standardized prior to conducting mediation analysis.

\section{Follow-up analyses}

Inclusion of outliers. Our data were reanalyzed after retaining both the identified outliers

Potential confounds: impact of sex differences, age, IQ, ICV, psychopathologies, and prescribed medications: Sex differences in irritability scores were determined used two-sample $t$ test. Correlation analyses were conducted to determine associations between ARI scores and age, IQ, measures of psychopathology (via Conners ADHD scale, ICU scale, MFQ scale, and GAD subscale), and current medication status (antipsychotic medications $[N=12]$, Selective Serotonin Reuptake Inhibitors [SSRIs; $N=22$ ], and stimulants [ $N=24]$ ). Stepwise multiple regression analyses were conducted to evaluate whether potential confounds [i.e., sex, age, IQ, ICV, measures of psychopathology, prescribed medications (scored 1 for "yes" or 0 for "no")] and hemispheric mean CV of identified networks significantly predicted irritability scores. To deal with multicollinearity, the regression analyses were performed for each measure of psychopathology separately.

Group Differences in cortical thickness and cortical surface area: All the main analyses (i.e., MANCOVA) performed for CV were repeated for cortical thickness and cortical surface area. For cortical thickness analysis, the potential covariates were sex, age, and IQ, whereas for cortical surface area analysis, the potential covariates were sex, age, IQ, and ICV.

Group differences in subcortical volume: All the main analyses (i.e., MANCOVA) performed for CV were repeated for subcortical volume for six subcortical areas i.e., thalamus, caudate, putamen, pallidum, hippocampus, and amygdala. For subcortical volume analysis, the potential covariates were sex, age, IQ, and ICV.

\section{RESULTS}

\section{Outlier detection}

Based on age and ARI scores, none of the participants were identified as outliers. However, two participants (one from each irritability group) had IQ more than 1.5 interquartile range above the upper quartile and were, therefore, excluded from further analysis.

\section{Demographics characteristics}

There were no group differences in $\operatorname{sex}\left(x^{2}=3.67, p=0.06\right)$ and age $\left(t(245)=-1.85, p=0.065 ; M_{\text {high irritability group }}=15.56, S D=1.83\right.$; $\left.M_{\text {low irritability group }}=15.12, S D=1.93\right)$. However, there were significant group differences in IQ $(t(245)=5.49, p<0.001$; $M_{\text {high irritability group }}=99.14, S D=12.13 ; M_{\text {low irritability group }}=107.64$, $S D=12.21)$ and ICV $\left(t(245)=2.45, p=0.01 ; M_{\text {high irritability group }}=\right.$ $1.47 \times 10^{6} \mathrm{~mm}^{3}, S D=0.15 \times 10^{6} ; M_{\text {low irritability group }}=1.52 \times 10^{6} \mathrm{~mm}^{3}$, $S D=0.15 \times 10^{6}$ ); see Table 1 . 
Table 1. Demographics.

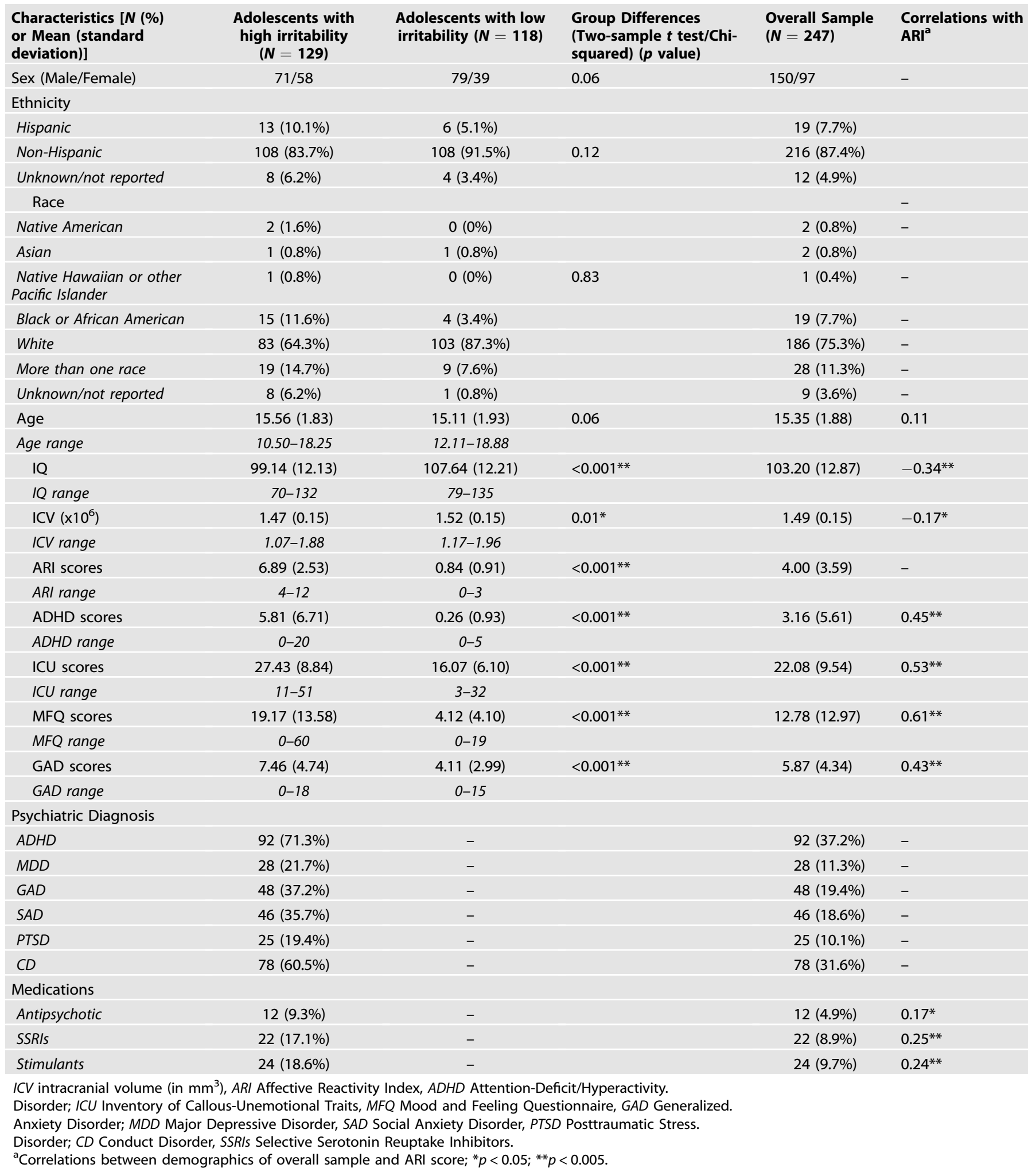

\section{Network-wise group differences in CV}

Our MANCOVA showed significant group differences in hemispheric-wise CV $\left[F(34,208)=1.55, p=0.03 ; p^{2}=0.20\right.$; Wilk's lambda $=0.80$ ]. There were significant differences bilaterally in CV for the control $\mathrm{B}$ network $(\mathrm{CBN} ;[F(1,241)=6.79 \& 8.59, p=0.01 \&$
$0.004, \mathrm{pn}^{2}=0.03 \& 0.03$, respectively for left and right hemisphere]; Fig. $1 \mathrm{~A})$ and default $\mathrm{B}$ network $(\mathrm{DBN} ;[F(1,241)=9.83 \& 7.22, p=$ $0.002 \& 0.01, \mathrm{p \eta}^{2}=0.04 \& 0.03$, respectively for left and right hemisphere]; Fig. 1B). For both networks, adolescents with high irritability showed lower CV than adolescents with low 


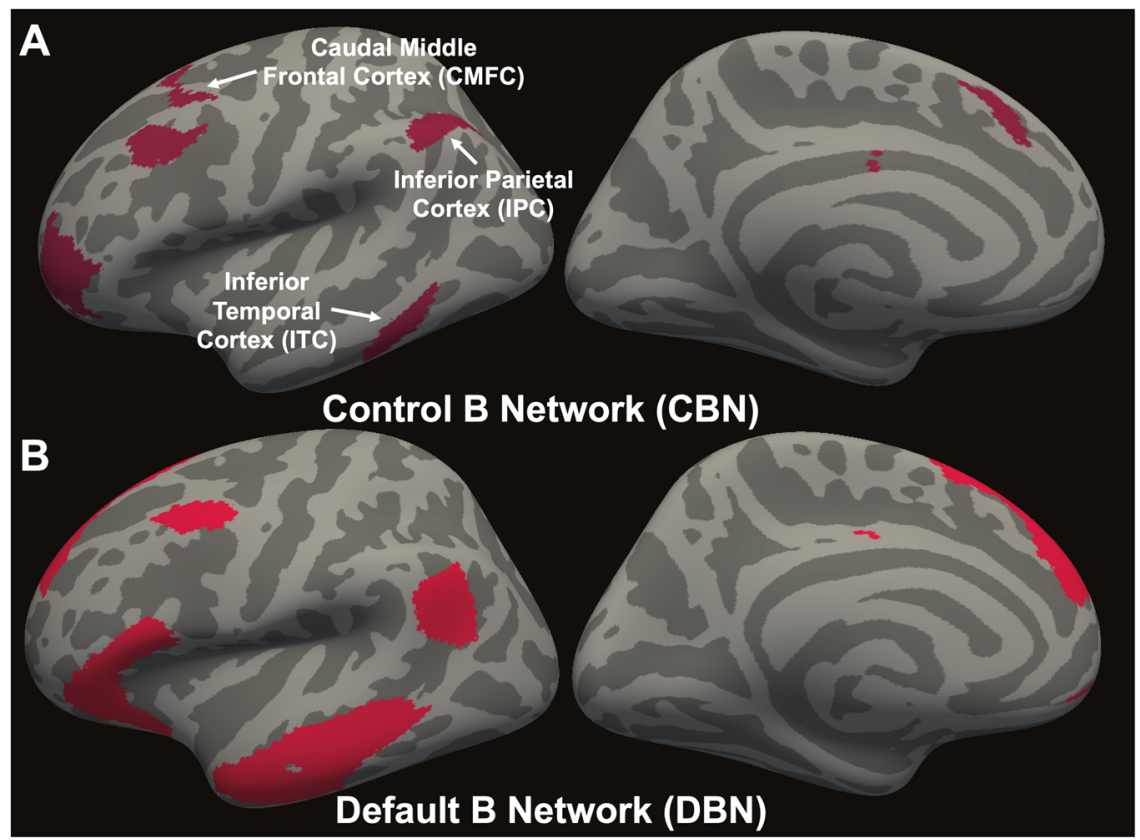

Fig. 1 Cortical networks showing significant differences in CV. Hemispheric mean CV of the control B network (CBN) (A) and default B network (DBN) (B) were significantly different between adolescents with high irritability and adolescents with low irritability (adolescents with high irritability < adolescents with low irritability).

irritability. No bilateral significant differences in CV were seen in the other networks at $p<0.05$ (see Table 2).

\section{Associations between mean CV and irritability symptom severity}

Our stepwise multiple regression analysis revealed a significant regression equation for irritability scores $[F(1,244)=11.91 ; p=$ 0.001 ]. Adjusted $R^{2}$ was 0.15 . Significant predictors for irritability scores were CBN CV (standardized $B=-0.21 ; p=0.001$ ) and IQ (standardized $B=-0.31 ; p<0.001$ ). DBN CV, sex, age, and ICV were nonsignificant predictors for irritability scores $\left(B_{\ln }=-0.07\right.$, $0.04,0.02 \& 0.03 ; p s=0.56,0.55,0.69, \& 0.68$, respectively).

\section{Associations between mean region-specific CV and irritability symptom severity}

Hemispheric-wise regions and a list of regions for which hemispheric mean CV was calculated for the CBN are summarized in Fig. 1A, Supplementary Table 1, and Supplementary Information. Within regions comprising the $\mathrm{CBN}$, the caudal middle frontal cortex (CMFC) and inferior temporal cortex (ITC) showed significant negative partial associations between $\mathrm{CV}$ and irritability symptom severity $(r=-0.16 \&-0.13, p=0.01 \& 0.04$, respectively; see Table 3). The inferior parietal cortex (IPC) also showed a strong trend of negative partial association between $\mathrm{CV}$ and irritability symptom severity $(r=-0.13, p=0.05$; see Table 3$)$.

\section{Mediation analysis: role of IQ in mediating the association between CV and irritability symptom severity}

Two separate standard mediation analyses were conducted to determine the role of IQ in mediating the association between hemispheric mean $\mathrm{CV}$ of $\mathrm{CBN}$ and its component regions (i.e., CMFC, ITC, and IPC that showed significant association with irritability) and irritability symptom severity.

Network-specific CV and irritability symptom severity. Greater CBN CV was significantly associated with greater IQ $(r=0.16, p=0.01)$ and lower levels of irritability $(r=-0.25, p<0.001)$. Greater IQ was also (independent of CBN CV) associated with lower levels of irritability $(r=-0.31, p<0.001)$. The mediation analysis revealed that while greater CBN CV was associated with lower levels of irritability (total effect, $c=-0.25, p<0.001$ ), this association did not disappear once IQ was included as an "intervening" factor (direct effect, $c^{\prime}=-0.20, p<0.001$ ). The bootstrap confidence interval for the indirect effect $(a b=-0.05 ;[-0.09-0.01]$ at $95 \%$ confidence interval) did not include zero. The percent mediation $\left(P_{M}\right)$ (i.e., percent of the total effect (c) accounted for by indirect effect (ab)) was $20 \%$. Findings indicate that IQ partially accounted for the association between the CBN CV and irritability symptom severity.

Region-specific CV and irritability symptom severity. CMFC and IPC CVs were not significantly associated with IQ $(r=0.10 \& 0.02, p=$ $0.10 \& 0.77$, respectively). Therefore, mediation analyses were not performed for CMFC and IPC. However, greater ITC CV was significantly associated with greater IQ $(r=0.13, p=0.03)$ and lower levels of irritability $(r=-0.20, p<0.005)$. Greater IQ was also (independent of ITC CV) associated with lower levels of irritability $(r=-0.32, p<0.001)$. The mediation analysis revealed that while greater ITC CV was associated with lower levels of irritability (total effect, $c=-0.20, p<0.005)$, this association did not disappear once IQ was included as an "intervening" factor (direct effect, $\left.c^{\prime}=-0.15, p<0.05\right)$. The bootstrap confidence interval for the indirect effect $(a b=-0.04 ;[-0.08-0.003]$ at $95 \%$ confidence interval) did not include zero. The percent mediation $\left(P_{M}\right)$ (i.e., percent of the total effect (c) accounted for by indirect effect $(a b))$ was $20 \%$. Findings indicate that IQ partially accounted for the association between the ITC CV and irritability symptom severity.

Figure 2 illustrates the path model (model 4) used to test the mediation effect of IQ on the association between CBN and ITC CVs and irritability (Fig. 2A, B).

\section{Follow-up analyses}

Inclusion of outliers. Reanalysis of our data after retaining both the identified outliers mostly mirrored our original results (see Supplementary section 2).

Potential confounds: impact of sex differences, age, IQ, ICV, psychopathologies, and prescribed medications: There were 
Table 2. Differences in Cortical Volume (CV): Adolescents with high irritability vs. adolescents with low irritability).

MANCOVA for CV (covariates: age, sex, IQ, and ICV)

\begin{tabular}{|c|c|c|c|c|c|c|}
\hline \multirow[t]{2}{*}{ Networks $(N)$} & \multicolumn{2}{|c|}{$p$ values } & \multicolumn{2}{|c|}{$F$ value } & \multicolumn{2}{|c|}{ Effect size: $p^{2}$} \\
\hline & LH & RH & $L H$ & RH & $L H$ & $R H$ \\
\hline N1: Visual Peripheral (VPN) & $0.04^{\mathrm{a}}$ & 0.75 & 4.32 & 0.10 & 0.02 & 0.00 \\
\hline N3: Somatomotor A (SAN) & 0.57 & 0.17 & 0.32 & 1.87 & 0.00 & 0.01 \\
\hline N4: Somatomotor B (SBN) & 0.49 & 0.14 & 0.48 & 2.24 & 0.00 & 0.01 \\
\hline N5: Dorsal Attention A (DAAN) & 0.29 & $0.008^{\mathrm{a}}$ & 1.10 & 7.13 & 0.00 & 0.03 \\
\hline N7: Ventral Attention (VAN) & 0.37 & $0.03^{\mathrm{a}}$ & 0.80 & 5.05 & 0.00 & 0.02 \\
\hline N8: Salience (SN) & $0.03^{a}$ & 0.43 & 4.60 & 0.61 & 0.02 & 0.00 \\
\hline N9: Limbic A (LAN) & $0.02^{\mathrm{a}}$ & 0.42 & 5.75 & 0.66 & 0.02 & 0.00 \\
\hline N10: Limbic B (LBN) & 0.10 & 0.05 & 2.69 & 3.77 & 0.01 & 0.01 \\
\hline N11: Control C (CCN) & 0.26 & 0.73 & 1.26 & 0.11 & 0.00 & 0.00 \\
\hline N15: Default C (DCN) & 0.12 & 0.37 & 2.42 & 0.80 & 0.01 & 0.00 \\
\hline N16: Default A (DAN) & $0.03^{\mathrm{a}}$ & 0.05 & 5.01 & 3.78 & 0.02 & 0.01 \\
\hline N17: Default B (DBN) & $0.002^{a}$ & $0.01^{\mathrm{a}}$ & 9.83 & 7.22 & 0.04 & 0.03 \\
\hline
\end{tabular}

MANCOVA Multivariate Analysis of Covariance, ICV intracranial volume. $L H / R H$ Left/Right Hemisphere.

${ }^{a} p<0.05$ (adolescents with high irritability < adolescents with low irritability).

Networks showing significant bilateral differences are bolded.

Table 3. Pearson's partial correlation (covariates: sex, age, IQ, and ICV) between region-specific mean cortical volume (CV) within the Control B Network (CBN) and irritability symptom severity.

\begin{tabular}{|c|c|c|c|c|}
\hline Network & Regions & Correlation ( $r$ ) & p-values & $95 \% \mathrm{Cl}$ \\
\hline \multirow[t]{6}{*}{ Control B Network (CBN) } & Superior Frontal Cortex (SFC) & 0.10 & 0.14 & {$\left[\begin{array}{ll}-0.03 & 0.22\end{array}\right]$} \\
\hline & Caudal Middle Frontal Cortex (CMFC) & -0.16 & $0.01 *$ & {$[-0.28-0.03]$} \\
\hline & Rostral Middle Frontal Cortex (RMFC) & -0.07 & 0.25 & {$\left[\begin{array}{lll}-0.20 & 0.05\end{array}\right]$} \\
\hline & Inferior Frontal Cortex (IFC) & 0.11 & 0.08 & {$\left[\begin{array}{lll}-0.01 & 0.24\end{array}\right]$} \\
\hline & Inferior Temporal Cortex (ITC) & -0.13 & $0.04 *$ & {$[-0.25-0.00]$} \\
\hline & Posterior Cingulate Cortex (PCC) & 0.02 & 0.79 & {$\left[\begin{array}{ll}-0.11 & 0.14\end{array}\right]$} \\
\hline
\end{tabular}

Cl Confidence Intervals; * $p<0.05$.

Regions showing significant or trend towards significant correlation are bolded.

Bolded numbers in the table indicate significant associations.

significant sex differences in irritability scores $(t(245)=-2.79$, $\left.p<0.01 ; M_{\text {males }}=3.49, S D=3.34 ; M_{\text {females }}=4.78, S D=3.83\right)$. Irritability scores were not correlated with age $(r=0.11, p>$ $0.05)$ but were negatively correlated with IQ and ICV $(r=$ $-0.34 \&-0.17$, ps $<0.05$; see Table 1 ). As expected, irritability scores were positively associated with measures of psychopathologies and prescribed medications $(r=0.17-0.61$, $p s<0.05$; see Table 1. Our stepwise multiple regression analyses revealed that $C B N C V$ (standardized $B$ ranges between -0.11 and -0.14 , $p s \leq 0.05$ ) was still one of the significant predictors of irritability scores even after including demographics characteristics (sex, age, and IQ), ICV, measures of psychopathologies, and prescribed medications as independent variables in the model (see Section 3 in Supplementary Material).
Group differences in cortical thickness and cortical surface area: Our MANCOVA showed nonsignificant group differences in cortical thickness $\left[F(34,209)=0.68, p=0.91 ; \mathrm{pn}^{2}=0.10\right]$ and cortical surface area $\left[F(34,208)=1.41, p=0.08 ; \mathrm{pn}^{2}=0.19\right]$.

Group differences in SCV: Our MANCOVA showed significant group differences in $\operatorname{SCV}\left[F(12,230)=2.24, p=0.01 ; \mathrm{p \eta}^{2}=0.10\right]$. No bilateral significant differences in SCV $[F(1,247)=0.00-5.58$, $\left.p=0.02-0.96, \mathrm{pn}^{2}=0.00-0.02\right]$ were seen in any of the subcortical structures at $p<0.05$ (Table 4). However, there was a strong trend showing bilateral differences in SCV of the amygdala (left amygdala: $F(1,247)=3.76, p=0.05$ and right amygdala: $F(1,247)=4.12, p=$ $0.04)$. The amygdala also showed a significant negative partial association between hemispheric mean SCV and irritability symptom severity $(r=-0.14, p=0.03)$. 
A

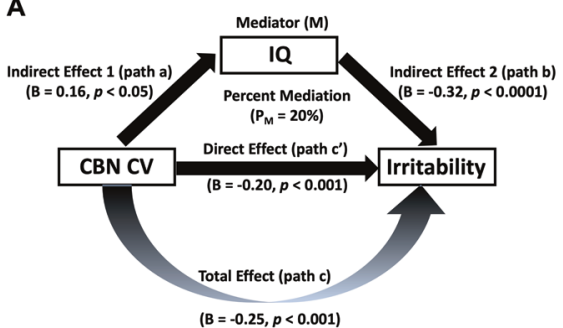

B

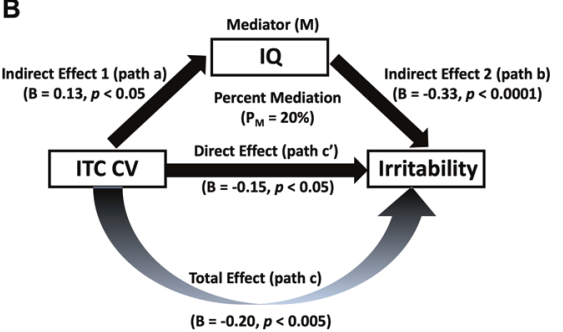

Fig. 2 Role of IQ in mediating the association of hemispheric mean CV of the control B network (CBN) and its component region (i.e., inferior temporal cortex [ITC]) with irritability symptom severity. IQ partially accounted for the association of both CBN and ITC CVs with irritability symptom severity (Figs A-B, respectively).

Table 4. Differences in Sub-Cortical Volume (SCV) (adolescents with high irritability vs. adolescents with low irritability).

\begin{tabular}{|c|c|c|c|c|c|c|}
\hline \multirow[t]{2}{*}{ Regions } & \multicolumn{2}{|c|}{$p$ values } & \multicolumn{2}{|c|}{$F$ value } & \multicolumn{2}{|c|}{$\begin{array}{l}\text { Effect Size: } \\
\text { pn }^{2}\end{array}$} \\
\hline & LH & $\mathbf{R H}$ & $L H$ & $R H$ & $L H$ & $\mathbf{R H}$ \\
\hline Thalamus & $0.02^{\mathrm{a}}$ & 0.51 & 5.58 & 0.44 & 0.02 & 0.00 \\
\hline Caudate & 0.30 & 0.96 & 1.07 & 0.00 & 0.00 & 0.00 \\
\hline Putamen & 0.40 & 0.06 & 0.71 & 3.54 & 0.00 & 0.01 \\
\hline Pallidum & 0.25 & 0.63 & 1.32 & 0.23 & 0.00 & 0.00 \\
\hline Hippocampus & 0.06 & 0.45 & 3.58 & 0.56 & 0.01 & 0.00 \\
\hline Amygdala & 0.05 & $0.04^{\mathrm{a}}$ & 3.76 & 4.12 & 0.01 & 0.02 \\
\hline
\end{tabular}

MANCOVA Multivariate Analysis of Covariance, ICV intracranial volume. $L H / R H$ Left/Right Hemisphere.

${ }^{a} p<0.05$ (adolescents with high irritability $<$ adolescents with low irritability).

\section{DISCUSSION}

The goals of the current study were to identify the following: (i) networks showing CV differences in adolescents with high irritability relative to adolescents with low irritability; (ii) the extent to which network-wise CV and their component regions predicted irritability symptom severity; and (iii) the extent to which IQ mediated the association between CV of identified networks/regions and irritability symptom severity. Our data indicated that, relative to participants with low irritability, adolescents with high irritability had reduced CV within the control $B$ and the default $B$ networks (CBN and $D B N)$ and reduced bilateral SCV within the amygdala. There was a negative association of CBN CV (and most of its regions, specifically the caudal middle frontal cortices, inferior temporal cortex, and inferior parietal cortex) and amygdala SCV with irritability symptom severity. Moreover, IQ partially mediated the association between the CBN CV and irritability symptom severity.

Regarding the direction of the cortical structure-irritability associations, previous studies have been inconsistent with respect to whether irritability is associated with greater cortical volume/ cortical thickening $[12,13]$ or decreased grey matter volume/ cortical thinning [14-17]. Our findings are clearly consistent with those studies associating irritability (or closely related constructs) with decreased grey matter volume/cortical thinning [14-17]. We note that these studies, as well as ours, differ from Dennis et al. 2019 and Pagliaccio et al. 2018 regarding participant age [12, 13]. Specifically, the two reports of associating irritability with greater cortical volume/cortical thickening involved relatively young participants (mean age $=11.5$ years [12]; mean age $=4.47$ years [13]). In contrast, the mean age of participants with irritability in the current study was 15.35 years, similar to that in Adleman et al. (14.2 years) [15], Chaarani et al. (14.5 years) [17], Gold et al.
(14.6 years) [16], and Jirsaraie et al. (16.2 years) [14]. Moreover, prior work has indicated that mean cortical thickness decreases while mean cortical surface area increases with age (and CV is more closely associated with cortical surface area [40]) and both cortical thickness and cortical surface area follow a linear trend [48]. However, mean CV follows either a linear or a quadratic trend, depending on regions of interest [48]. Interestingly, overall, maxima or minima was observed at roughly 12 years of age when a quadratic function was fit to best illustrate the age-related changes [48]. The observed cut-off age of roughly 12 years here is approximately consistent with the mean age of 11.5 years in the study associating irritability with greater cortical volume/cortical thickening [12]. In short, we believe the current data (mean age = 15.35 years), together with previous work on different CV/ thickness/surface area-age trajectories across different age groups, suggests that, for relatively older adolescents, irritability is associated with decreased grey matter volume/cortical thinning in relevant regions. Future research will benefit from longitudinal data collected across a wide age range in adolescents.

Consistent with our a priori hypotheses, there were significant group differences within the control and default mode networks (specifically, significantly decreased CBN and DBN volumes for the adolescents with high irritability as compared to adolescents with low irritability). While there were group differences in network volumes and IQ, IQ could not account for these differences-given the results of our stepwise multiple regression with IQ as a covariate. To our knowledge, these results are the first to show network-wise structural alterations (at a whole-brain level) associated with elevated irritability. Prior structural MRI studies have mostly reported region-specific structural alterations associated with irritability [12-14, 17]. Notably, our data are consistent with prior functional work suggesting that dysfunction in components of the CBN mediating inhibitory control is associated with increased irritability [7-9, 49]. In addition, some connectivity analyses of resting-state functional MRI data have examined associations of network-level connectivity (particularly in the default-mode network) with constructs that are closely related to irritability. Specifically, the negative mood state of anger has been associated with connectivity alterations within the default-mode network [50,51]. Patients with bipolar or borderline personality disorders, which are characterized by high levels of anger, have shown both abnormal functional $[52,53]$ and structural organization (e.g., reduced grey matter) [54] within the default-mode network. Moreover, prior functional MRI work has associated irritability with atypical functioning in components of the default-mode network $[10,11]$. Thus, despite the lack of prior network-wise structural MRI work on irritability, the current results are consistent with previous network-wise functional and structural MRI work, particularly on the default-mode network and constructs that are closely related to irritability (e.g., anger or bipolar and borderline personality disorder).

In contrast to our hypotheses, there were no significant group differences within the limbic network. Given strong empirical claims that irritability is associated with atypical emotional 
responding $[3,55]$, this was unexpected. Moreover, none of the subcortical structures showed bilateral group differences in subcortical volume. Future work will be needed to explore these issues further. Also, there were no significant group differences in cortical thickness and cortical surface area, but CV showed significant group differences. As mentioned before, both the cortical thickness and cortical surface area measurements influence CV measurements [40]. Therefore, we observed significant group differences in CV only as the joint analysis of thickness and surface area in terms of CV increases the power to simultaneously quantify the effects of thickness and surface area.

$\mathrm{CV}$ within the CBN, but not the DBN, was negatively associated with irritability symptom severity. With respect to specific anatomical regions within the $\mathrm{CBN}$, there were significant negative associations between $\mathrm{CV}$ of the caudal middle frontal cortex, inferior temporal cortex, and the inferior parietal cortex and irritability (see Table 3). These findings were consistent with previous functional neuroimaging studies of irritability which have associated atypical recruitment of regions within the middle frontal cortex and inferior parietal lobule with irritability $[49,56]$. In a review paper on the adult population, decreased grey matter volume/cortical thinning within the middle frontal gyrus and a large proportion of the temporal cortex has been found in patients with bipolar disorder [57]. In sum, prior functional and structural findings on irritability support the currently observed 'region-specific' associations of irritability.

In line with our predictions, IQ at-least partially mediated the relationship between pathophysiology and irritability. Notably, this mediation was true only for the relationship between CBN CV (and its component region ITC) and irritability. Given the crosssectional nature of the current study, the mediation findings are purely based on correlation analyses and should not be interpreted or viewed as "causal" in nature. These results are consistent with our predictions generated from previous work indicating that IQ (i.e., the overall level of general cognitive and intellectual functioning, and faster processing speeds/lower reaction time) is inversely associated with the risk for the expression of a variety of psychiatric disorders, including ADHD [24], depression [58], and, importantly for the current study, anger as a response to stress [59]. We hypothesize that this reflects a role for IQ in fostering the development of executive function (e.g., working memory and internal locus of control) $[27-30,60]$ that reduces the probability that the individual will express an underlying neuro-biological risk factor in symptomatology. Prior work has shown that $\mathrm{IQ} /$ general intelligence was significantly associated with both-at-least $80 \%$ executive function indices $[28,61]$, as well as measures of locus of control [29, 62]. Both executive function and a more internal locus of control have also been associated with reduced psychological distress $[30,63]$ and aggression [64-66]. We assume that the negative association between CBN CV and irritability reflects poorer control over the behavioral expressions of irritability. As such, an increased ability to control behavior (executive function more generally) and an increased sense that the individual can control their behavior (locus of control) may protect the individual from developing the irritability symptom profile. Future work will be necessary to examine these speculations in more detail. Additionally, the cortical association area 36 that involves portions of the lateral ITC (and medial fusiform gyrus) has a strong influence on cognitive and visual recognition abilities, mostly assessed with general IQ testing [22]. The association of structural deficits within inferior temporal regions with both reduced cognitive/recognition abilities [22] and elevated levels of irritability [14] support the notion that IQ might be playing a mediating role between reduced CV within the ITC and irritability symptom severity. Moreover, temporal regions, by themselves, are not only crucial for affect regulation [67] but may also modulate aggression via activating cortical and subcortical emotional response system through its connections with the amygdala and hypothalamus [68]. In sum, structural alteration in such regions may be critical for elevated levels of irritability [14].

The current findings must be viewed considering the following three caveats. First, there were group differences in $\mathrm{IQ}$ and ICV. However, mitigating this concern, the effect of IQ and ICV was controlled for within our group analyses. Second, networks of interest were constrained only if there were bilateral significant differences in morphometric measures. Therefore, the hemispheric laterality was not analyzed in the current study. Third, the participants with high irritability showed significant psychiatric comorbidities and some were receiving psychiatric medications. However, the follow-up analyses that we conducted with psychiatric comorbidities and psychiatric medications as additional predictors in our multiple regression analyses mirrored our results. This suggested that psychiatric comorbidities and psychiatric medications did not confound the current results and that CBN CV was still one of the significant predictors of irritability.

In summary, we found significantly lower CV within the control and default-mode networks in adolescents with high irritability as compared to adolescents with low irritability. Moreover, control network CV showed a significant negative association with irritability. The association between control network CV and irritability was partially mediated by IQ. The current findings enhance our fundamental understanding of cortical structure and irritability at the level of large-scale brain networks that have been specified by considerable prior functional and structural MRI work [69-74], and further suggest core structural impairments related to the expression of irritability.

\section{REFERENCES}

1. Camacho MC, Wakschlag LS, Perlman SB. Early Childhood Irritability: Using a neurodevelopmental framework to inform clinical understanding. In: Roy A, Brotman M, Leibenluft E (eds). Irritability in Pediatric Psychopathology. Oxford University Press, 2018.

2. Wakschlag LS, Perlman SB, Blair RJ, Leibenluft E, Briggs-Gowan MJ, Pine DS. The neurodevelopmental basis of early childhood disruptive behavior: Irritable and callous phenotypes as exemplars. Am J Psychiatry. 2018;175:114-30.

3. Blair RJR. Considering anger from a cognitive neuroscience perspective. Wiley Interdiscip Rev Cogn Sci. 2012;3:65-74.

4. Leibenluft E. Pediatric Irritability: A Systems Neuroscience Approach. Trends Cogn Sci. 2017;21:277-89.

5. Vidal-Ribas P, Brotman MA, Valdivieso I, Leibenluft E, Stringaris A. The Status of Irritability in Psychiatry: A Conceptual and Quantitative Review. J Am Acad Child Adolesc Psychiatry. 2016;55:556-70.

6. Yeo BT, Krienen FM, Sepulcre J, Sabuncu MR, Lashkari D, Hollinshead M, et al. The organization of the human cerebral cortex estimated by intrinsic functional connectivity. J Neurophysiol. 2011;106:1125-65.

7. Deveney CM, Briggs-Gowan MJ, Pagliaccio D, Estabrook CR, Zobel E, Burns JL, et al. Temporally sensitive neural measures of inhibition in preschool children across a spectrum of irritability. Dev Psychobiol. 2019;61:216-27.

8. Fishburn FA, Hlutkowsky CO, Bemis LM, Huppert TJ, Wakschlag LS, Perlman SB. Irritability uniquely predicts prefrontal cortex activation during preschool inhibitory control among all temperament domains: A LASSO approach. Neuroimage. 2019;184:68-77.

9. Zisner A, Beauchaine TP. Neural substrates of trait impulsivity, anhedonia, and irritability: Mechanisms of heterotypic comorbidity between externalizing disorders and unipolar depression. Dev Psychopathol. 2016;28:1177-208.

10. Crum Kl, Hwang S, Blair KS, Aloi JM, Meffert H, White SF, et al. Interaction of irritability and anxiety on emotional responding and emotion regulation: a functional MRI study. Psychol Med. 2020;25:1-11.

11. Stoddard J, Tseng WL, Kim P, Chen G, Yi J, Donahue L, et al. Association of irritability and anxiety with the neural mechanisms of implicit face emotion processing in youths with psychopathology. JAMA Psychiatry. 2017;74:95-103.

12. Dennis EL, Humphreys KL, King LS, Thompson PM, Gotlib IH. Irritability and brain volume in adolescents: Cross-sectional and longitudinal associations. Soc Cogn Affect Neurosci. 2019;14:687-98.

13. Pagliaccio D, Pine DS, Barch DM, Luby JL, Leibenluft E. Irritability Trajectories, Cortical Thickness, and Clinical Outcomes in a Sample Enriched for Preschool Depression. J Am Acad Child Adolesc Psychiatry. 2018;57:336-42. 
14. Jirsaraie RJ, Kaczkurkin AN, Rush S, Piiwia K, Adebimpe A, Bassett DS, et al. Accelerated cortical thinning within structural brain networks is associated with irritability in youth. Neuropsychopharmacology. 2019;44:2254-62.

15. Adleman NE, Fromm SJ, Razdan V, Kayser R, Dickstein DP, Brotman MA, et al. Cross-sectional and longitudinal abnormalities in brain structure in children with severe mood dysregulation or bipolar disorder. J Child Psychol Psychiatry Allied Discip. 2012;53:1149-56.

16. Gold AL, Brotman MA, Adleman NE, Lever SN, Steuber ER, Fromm SJ, et al. Comparing Brain Morphometry Across Multiple Childhood Psychiatric Disorders. J Am Acad Child Adolesc Psychiatry. 2016;55:1027-1037.e3.

17. Chaarani B, Kan K-J, Mackey S, Spechler PA, Potter A, Banaschewski T, et al. Neural Correlates of Adolescent Irritability and Its Comorbidity With Psychiatric Disorders. J Am Acad Child Adolesc Psychiatry. 2020;59:1371-9.

18. Winkler AM, Greve DN, Bjuland KJ, Nichols TE, Sabuncu MR, Håberg AK, et al. Joint Analysis of Cortical Area and Thickness as a Replacement for the Analysis of the Volume of the Cerebral Cortex. Cereb cortex. 2018;28:738-49.

19. Paulus MP, Squeglia LM, Bagot K, Jacobus J, Kuplicki R, Breslin FJ, et al. Screen media activity and brain structure in youth: Evidence for diverse structural correlation networks from the ABCD study. Neuroimage. 2019;185:140-53.

20. Chen J, Tam A, Kebets V, Orban C, Ooi LQR, Marek S, et al. Shared and unique brain network features predict cognition, personality and mental health in childhood. bioRxiv 2020: 2020.06.24.168724.

21. Bajaj S, Raikes A, Smith R, Dailey NS, Alkozei A, Vanuk JR, et al. The Relationship Between General Intelligence and Cortical Structure in Healthy Individuals. Neuroscience. 2018;388:36-44.

22. Narr KL, Woods RP, Thompson PM, Szeszko P, Robinson D, Dimtcheva T, et al Relationships between IQ and regional cortical gray matter thickness in healthy adults. Cereb Cortex. 2007;17:2163-71.

23. Menary K, Collins PF, Porter JN, Muetzel R, Olson EA, Kumar V, et al. Associations between cortical thickness and general intelligence in children, adolescents and young adults. Intelligence. 2013;41:597-606.

24. Melby L, Indredavik MS, Løhaugen G, Brubakk AM, Skranes J, Vik T. Is there an association between full IQ score and mental health problems in young adults? A study with a convenience sample. BMC Psychol. 2020;8:7.

25. Gale CR, Batty GD, Tynelius P, Deary IJ, Rasmussen F. Intelligence in early adulthood and subsequent hospitalisation and admission rates for the whole range of mental disorders: longitudinal study of 1,049,663 men. Epidemiology. 2010;21:70-77.

26. Wraw C, Deary IJ, Der G, Gale CR. Intelligence in youth and mental health at age 50. Intelligence. 2016;58:69-79.

27. Duan X, Wei S, Wang G, Shi J. The relationship between executive functions and intelligence on 11- to 12-year- old children. Psychol Test Assess Model. 2010;52:419-31.

28. Arffa S. The relationship of intelligence to executive function and non-executive function measures in a sample of average, above average, and gifted youth. Arch Clin Neuropsychol. 2007;22:969-78.

29. Brown RT. Locus of Control and its Relationship to Intelligence and Achievement. Psychol Rep. 1980;46:1249-50.

30. Gale CR, Batty GD, Deary IJ. Locus of control at age 10 years and health outcomes and behaviors at age 30 years: The 1970 british cohort study. Psychosom Med. 2008;70:397-403.

31. Mulraney M, Melvin G, Tonge B. Brief report: Can irritability act as a marker of psychopathology? J Adolesc. 2014;37:419-23.

32. Stringaris A, Goodman R, Ferdinando S, Razdan V, Muhrer E, Leibenluft E, et al. The Affective Reactivity Index: A concise irritability scale for clinical and research settings. J Child Psychol Psychiatry Allied Discip. 2012;53:1109-17.

33. Wechsler D. Wechsler Abbreviated Scale of Intelligence-Second Edition (WASI-II). NCS Pearson: San Antonio, TX, 2011.

34. McCrimmon AW, Smith AD. Review of the Wechsler Abbreviated Scale of Intelligence, Second Edition (WASI-II). J Psychoeduc Assess. 2013;31:337-41.

35. Irby SM, Floyd RG. Test Review: Wechsler Abbreviated Scale of Intelligence, Second Edition. Can J Sch Psychol. 2013;28:295-9.

36. Conners CK. Conners 3rd edition manual. Multi-Health Systems: Toronto, Ontario, Canada, 2008.

37. Essau CA, Sasagawa S, Frick PJ. Callous-unemotional traits in a community sample of adolescents. Assessment. 2006;13:454-69.

38. Angold A, Costello EJ, Messer SC, Pickles A. Development of a short questionnaire for use in epidemiological studies of depression in children and adolescents. Int J Methods Psychiatr Res. 1995;5:237-49.

39. Birmaher B, Brent DA, Chiappetta L, Bridge J, Monga S, Baugher M. Psychometric properties of the screen for child anxiety related emotional disorders (SCARED): A replication study. J Am Acad Child Adolesc Psychiatry. 1999;38:1230-6.

40. Winkler AM, Kochunov P, Blangero J, Almasy L, Zilles K, Fox PT, et al. Cortical thickness or grey matter volume? The importance of selecting the phenotype for imaging genetics studies. Neuroimage. 2010;53:1135-46.
41. Fischl B, Sereno MI, Dale AM. Cortical surface-based analysis. II: Inflation, flattening, and a surface-based coordinate system. Neuroimage. 1999;9:195-207.

42. Dale AM, Fischl B, Sereno MI. Cortical surface-based analysis. I. Segmentation and surface reconstruction. Neuroimage. 1999;9:179-94.

43. Desikan R, Ségonne F, Fischl B, Quinn B, Dickerson B, Blacker D, et al. An automated labeling system for subdividing the human cerebral cortex on MRI scans into gyral based regions of interest. Neuroimage. 2006;31:968-80.

44. Fischl B, van der Kouwe A, Destrieux C, Halgren E, Segonne F, Salat DH, et al. Automatically parcellating the human cerebral cortex. Cereb Cortex. 2004;14:11-22.

45. Bajaj S, Dailey NS, Rosso IM, Rauch SL, Killgore WDS. Time-dependent differences in cortical measures and their associations with behavioral measures following mild traumatic brain injury. Hum Brain Mapp 2018;39:1886-97.

46. George D, Mallery P. SPSS for Windows step by step: a simple guide and reference, 17.0 update. Allyn \& Bacon: Boston, MA, 2010.

47. Hayes AF. Introduction to mediation, moderation, and conditional process analysis: A regression-based approach. 2nd ed. Guilford Press: New York, NY, 2018.

48. Sussman D, Leung RC, Chakravarty MM, Lerch JP, Taylor MJ. The developing human brain: age-related changes in cortical, subcortical, and cerebellar anatomy. Brain Behav. 2016;6:e00457.

49. Tseng WL, Deveney CM, Stoddard J, Kircanski K, Frackman AE, Yi JY, et al. Brain mechanisms of attention orienting following frustration: Associations with irritability and age in youths. Am J Psychiatry. 2019;176:67-76.

50. Dong GH, Li H, Wang LX, Potenza MN. The correlation between mood states and functional connectivity within the default mode network can differentiate Internet gaming disorder from healthy controls. Prog Neuropsychopharmacol Biol Psychiatry. 2017;77:185-93.

51. Luo Y, Kong F, Qi S, You X, Huang X. Resting-state functional connectivity of the default mode network associated with happiness. Soc Cogn Affect Neurosci. 2016;11:516-24.

52. Öngür D, Lundy M, Greenhouse I, Shinn AK, Menon V, Cohen BM, et al. Default mode network abnormalities in bipolar disorder and schizophrenia. Psychiatry Res - Neuroimaging. 2010;183:59-68.

53. Zovetti N, Rossetti MG, Perlini C, Maggioni E, Bontempi P, Bellani M, et al Default mode network activity in bipolar disorder. Epidemiol Psychiatr Sci 2020;29:e166.

54. Yang $X, H u$ L, Zeng J, Tan $Y$, Cheng $B$. Default mode network and frontolimbic gray matter abnormalities in patients with borderline personality disorder: A voxel-based meta-analysis. Sci Rep. 2016;6:34247.

55. Brotman MA, Kircanski K, Leibenluft E. Irritability in Children and Adolescents. Annu Rev Clin Psychol. 2017;13:317-41.

56. Kircanski K, White LK, Tseng WL, Wiggins JL, Frank HR, Sequeira S, et al. A latent variable approach to differentiating neural mechanisms of irritability and anxiety in youth. JAMA Psychiatry. 2018;75:631-9.

57. Hanford LC, Nazarov A, Hall GB, Sassi RB. Cortical thickness in bipolar disorder: A systematic review. Bipolar Disord. 2016;18:4-18.

58. Gale CR, Deary IJ, Boyle SH, Barefoot J, Mortensen LH, Batty GD. Cognitive ability in early adulthood and risk of 5 specific psychiatric disorders in middle age: The Vietnam Experience Study. Arch Gen Psychiatry. 2008;65:1410-8.

59. Jung $\mathrm{YH}$, Shin NY, Jang JH, Lee WJ, Lee D, Choi Y, et al. Relationships among stress, emotional intelligence, cognitive intelligence, and cytokines. Med. 2019;98:e15345.

60. Colom R, Abad FJ, Quiroga MÁ, Shih PC, Flores-Mendoza C. Working memory and intelligence are highly related constructs, but why? Intelligence. 2008;36: 584-606.

61. Mahone EM, Hagelthorn KM, Cutting LE, Schuerholz LJ, Pelletier SF, Rawlins C, et al. Effects of IQ on executive function measures in children with ADHD. Child Neuropsychol a J Norm Abnorm Dev Child Adolesc. 2002;8:52-65.

62. Findley MJ, Cooper HM. Locus of control and academic achievement: A literature review. J Pers Soc Psychol. 1983;44:419-27.

63. Zebdi R, Goyet L, Pinabiaux C, Guellaï B. Psychological disorders and ecological factors affect the development of executive functions: Some perspectives. Front Psychiatry. 2016;7:195.

64. Ellis ML, Weiss B, Lochman JE. Executive functions in children: Association with aggressive behavior and appraisal processing. J Abnorm Child Psychol. 2009;37:945-56.

65. Breet $L$, Myburgh C, Poggenpoel M. The relationship between the perception of own locus of control and aggression of adolescent boys. South Afr J Educ. 2010;30:511-26.

66. Deming AM, Lochman JE. The relation of locus of control, anger, and impulsivity to boys'. aggressive Behav Behav Disord. 2008;33:108-19.

67. Morawetz C, Bode S, Baudewig J, Jacobs AM, Heekeren HR. Neural representation of emotion regulation goals. Hum Brain Mapp. 2016;37:600-20.

68. Potegal M. Temporal and frontal lobe initiation and regulation of the top-down escalation of anger and aggression. Behav Brain Res. 2012;231:386-95. 
10

69. Fjell AM, Sneve MH, Grydeland $H$, Storsve AB, Amlien IK, Yendiki A, et al. Relationship between structural and functional connectivity change across the adult lifespan: A longitudinal investigation. Hum Brain Mapp. 2017;38:561-73.

70. Oligschläger S, Huntenburg JM, Golchert J, Lauckner ME, Bonnen T, Margulies DS. Gradients of connectivity distance are anchored in primary cortex. Brain Struct Funct. 2017;222:2173-82.

71. Taylor PN, Forsyth R. Heterogeneity of trans-callosal structural connectivity and effects on resting state subnetwork integrity may underlie both wanted and unwanted effects of therapeutic corpus callostomy. Neurolmage Clin. 2016;12:341-7.

72. Palaniyappan L, Al-Radaideh A, Gowland PA, Liddle PF. Cortical thickness and formal thought disorder in schizophrenia: an ultra high-field networkbased morphometry study. Prog Neuro-Psychopharmacology Biol Psychiatry 2020;101:109911.

73. Sawczak CM, Barnett AJ, Cohn M, Bologna M. Increased cortical thickness in attentional networks in Parkinson's disease with minor hallucinations. Parkinsons Dis. 2019;2019:5351749.

74. Shafiei G, Markello RD, Makowski C, Talpalaru A, Kirschner M, Devenyi GA, et al. Spatial patterning of tissue volume loss in Schizophrenia reflects brain network architecture. Biol Psychiatry. 2020;87:727-35.

\section{ACKNOWLEDGEMENTS}

We would like to thank Ron Copsey, Kim VanHorn, Michael Wright, Mark Timm, Michelle Kelly, and Sarah Johnson for their contributions to data collection. This research was in part supported by the National Institute of Mental Health under award number K22-MH109558 (RJRB). The funders had no role in the design and conduct of the study; collection, management, analysis, and interpretation of the data; preparation, review, or approval of the manuscript; and decision to submit the manuscript for publication.

\section{AUTHOR CONTRIBUTIONS}

S.B. analyzed the data and wrote the initial draft. K.S.B. contributed to study design, data analysis, and writing of the manuscript. J.B.L., R.Z., A.M., A.S., and J.E. contributed to data analysis and writing of the manuscript. M.D., S.H., and E.L. provided their expertise in the field of psychiatry and contributed to the writing of the manuscript.
R.J.B. obtained the funding, supervised all aspects of the study, and contributed to the writing of the manuscript.

\section{COMPETING INTERESTS}

The authors declare no competing interests.

\section{ADDITIONAL INFORMATION}

Supplementary information The online version contains supplementary material available at https://doi.org/10.1038/s41398-021-01710-2.

Correspondence and requests for materials should be addressed to Sahil Bajaj.

Reprints and permission information is available at http://www.nature.com/ reprints

Publisher's note Springer Nature remains neutral with regard to jurisdictional claims in published maps and institutional affiliations.

(i) Open Access This article is licensed under a Creative Commons Cc Attribution 4.0 International License, which permits use, sharing, adaptation, distribution and reproduction in any medium or format, as long as you give appropriate credit to the original author(s) and the source, provide a link to the Creative Commons license, and indicate if changes were made. The images or other third party material in this article are included in the article's Creative Commons license, unless indicated otherwise in a credit line to the material. If material is not included in the article's Creative Commons license and your intended use is not permitted by statutory regulation or exceeds the permitted use, you will need to obtain permission directly from the copyright holder. To view a copy of this license, visit http://creativecommons. org/licenses/by/4.0/.

(c) The Author(s) 2021 